\title{
Analysis of a synthetic turbulence generation method for periodic configurations
}

\author{
C. Morsbach and M. Franke
}

\section{Introduction}

With increasingly available computational resources, scale-resolving simulations begin to become affordable for industrially relevant flows. While full Large Eddy Simulations (LES) may still be out of reach, the combination of Reynolds-Averaged Navier-Stokes (RANS) and LES methods is a promising approach. Both LES and zonal RANS-LES methods require the prescription of resolved velocity fluctuations at the inflow or the RANS-LES interfaces. Especially in turbomachinery applications, due to upstream blade rows or the combustor, the inflow is highly turbulent and the prediction of phenomena such as transition depends crucially on the correct representation of turbulent scales. To save computational resources, the solution is usually assumed to be periodic in one or two directions. One assumption is spanwise periodicity when blade profiles are computed. Specifically in a turbomachinery application, it is often reasonable to compute only the flow around a fraction of the blades and apply rotational periodicity to model the full wheel.

A method to obtain realistic velocity fluctuations is a precursor simulation, from which the turbulent flow field is extracted and prescribed at the inflow of the actual simulation. Since this is not feasible for general industrial applications, various methods have been devised to generate resolved velocity fluctuations from statistical quantities, such as the Reynolds stress tensor and the turbulent length scale [7]. Keating et al. [2] review the performance of different approaches of prescribing a proper turbulence field in plane channel flow. We implemented the synthetic turbulence generation method recently suggested by Shur et al. [5] in DLR's flow solver for turbomachinery applications TRACE [1]. The method is based on a Fourier reconstruction of the fluctuating velocity field using wave number vectors with uniformly distributed random directions.

C. Morsbach · M. Franke

Numerical Methods, Institute of Propulsion Technology, German Aerospace Center (DLR), e-mail:

christian.morsbachedlr.de 
By definition, turbulence generated by Shur's method is not periodic in a given direction. This leads to a violation of continuity at periodic boundaries of the computational domain and can, in turn, result in abnormal turbulence statistics downstream. Motivated by this deficiency, we propose a simple correction which restores periodicity. We will evaluate the performance of the correction with respect to statistical properties of turbulence such as Reynolds stresses, two-point correlations and energy spectra.

\section{Synthetic turbulence generator and periodic correction}

The synthetic turbulence generator is implemented as described in Shur et al. [5] Only the parts of the formulation which are important to understand the deficiency and proposed correction are stated here. For all details of the approach, the reader is referred to the original publication. The velocity fluctuations $\mathbf{v}^{\prime}$ at the location $\mathbf{r}$ and time $t$ are given by

$$
\mathbf{v}^{\prime}(\mathbf{r}, t)=2 \sqrt{\frac{3}{2}} \sum_{n=1}^{N} \sqrt{q^{n}}\left[\sigma^{n} \cos \left(k^{n} \mathbf{d}^{n} \cdot \mathbf{r}^{\prime}(\mathbf{r}, t)+\phi^{n}\right)\right] .
$$

The wave numbers $k^{n}$ and amplitudes $\sqrt{q^{n}}$ are determined from a modified vonKarman spectrum. The vectors $\mathbf{d}^{n}$ are uniformly distributed over a unit sphere. For the condition of vanishing divergence, $\sigma^{n} \perp \mathbf{d}^{n}$ is required and $\sigma^{n}$ is computed to be in the plane perpendicular to the respective $\mathbf{d}^{n}$ and rotated by a uniformly distributed angle in the interval $[0,2 \pi)$. Finally uniformly distributed random phases $\phi^{n}$ are selected. The spatiotemporal variation is determined by

$$
\mathbf{r}^{\prime}(\mathbf{r}, t)=\left(\begin{array}{c}
\frac{k_{e}}{k^{n}}\left(x-U_{0} t\right) \\
y \\
z
\end{array}\right)
$$

with the wave number $k_{e}$ of the most energetic eddies and the mean velocity $U_{0}$.

For the velocity signal to be periodic in a domain with lengths $L_{i}$, the relations

$$
k^{n} d_{i}^{n} L_{i}=2 \pi m_{i}^{n}, \quad i \in\{y, z\}
$$

with integer numbers $m_{i}^{n} \in \mathbb{Z}$ need to be fulfilled for every combination of wave number $k^{n}$ and random unit vector $\mathbf{d}^{n}$. In the original formulation of the method, this is generally not the case. Therefore, we propose a simple algorithm for each $k^{n}$ to ensure these conditions:

1. Find the largest $m_{y}^{n}$ for which $d_{y}^{n}<1$

2. Find the largest $m_{z}^{n}$ for which $\sqrt{\left(d_{y}^{n}\right)^{2}+\left(d_{z}^{n}\right)^{2}}<1$

3. Rescale $d_{i}^{n}=\frac{2 \pi m_{i}^{n}}{k^{n} L_{i}}$ for $i \in\{y, z\}$ 
4. Recompute $d_{x}=1-\sqrt{\left(d_{y}^{n}\right)^{2}+\left(d_{z}^{n}\right)^{2}}$

In other words, the $k_{i}^{n} d_{i}^{n} L_{i}$ are rounded to the closest multiple of $2 \pi$ with the restriction of the resulting vector being possible to scale to a length of 1 . For the wave number vectors $k^{n} \mathbf{d}^{n}$ this means that their $y$-and $z$-components are multiples of the minimum wavenumber corresponding to the domain size.

Fig. 1 shows the effect of this algorithm on the distribution of random vectors in terms of a probability density function (PDF) for each component of the vectors $\mathbf{d}^{n}$. For random vectors uniformly distributed on a sphere, the expected PDF would be a constant value of 0.5 as indicated by the dotted line. Both $y$ - and $z$-components show a peak at zero with a resulting depletion of the $x$-component. This is a result of the proposed rounding strategy for small wave numbers $k^{n}$.

Fig. 1 Probability density function for components of $\mathbf{d}^{n}$ after application of periodic correction in $y$ - and $z$-direction. The dotted line indicates the expected value for uniformly distributed random vectors on a sphere.

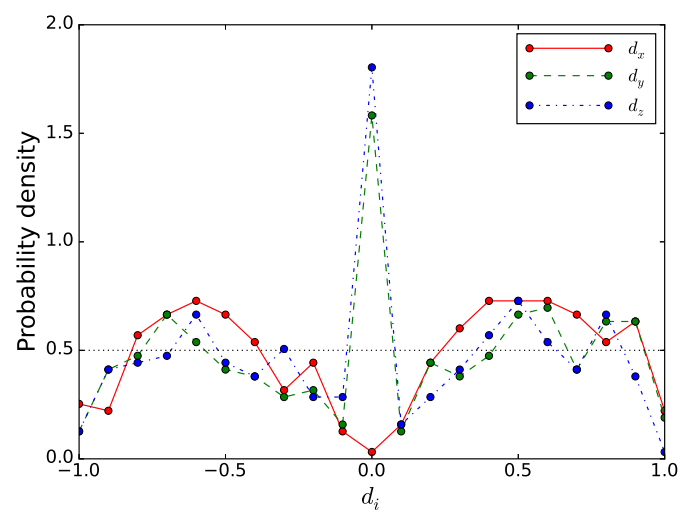

\section{Analysis of generated turbulence}

Spatially decaying homogeneous isotropic turbulence was computed to test the inflow boundary condition. The domain size is given in multiples of the turbulent length scale $L_{T}$ as $8 \pi \times 2 \pi \times 2 \pi$ on a mesh of $128 \times 32 \times 32$ cells. At the inlet, the velocity fluctuations obtained from the synthetic turbulence generator are superimposed on the constant bulk velocity $U_{0}$. At the outlet, a constant static pressure is specified using a non-reflecting boundary condition.

The filtered Navier-Stokes equations are discretised using a second-order accurate finite volume scheme applying MUSCL reconstruction with $\kappa=1 / 3$ [3]. A fraction of $10^{-3}$ of Roe's numerical flux [4] is added to a central flux to avoid oddeven decoupling. Time integration is performed using a third order accurate explicit Runge-Kutta method. The subgrid stresses are computed by a classic Smagorinsky model [6]. 

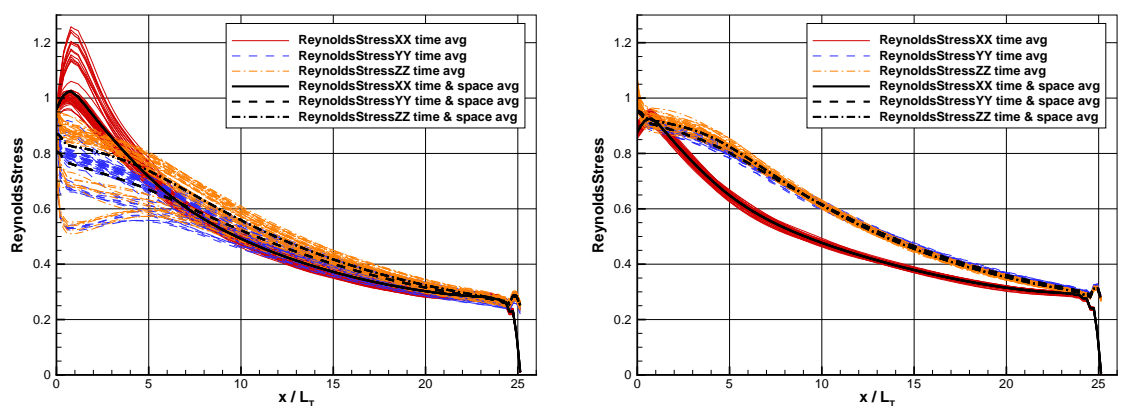

Fig. 2 Temporally and both temporally and spatially averaged decay of Reynolds stress tensor components for the original formulation (left) and periodic correction (right).
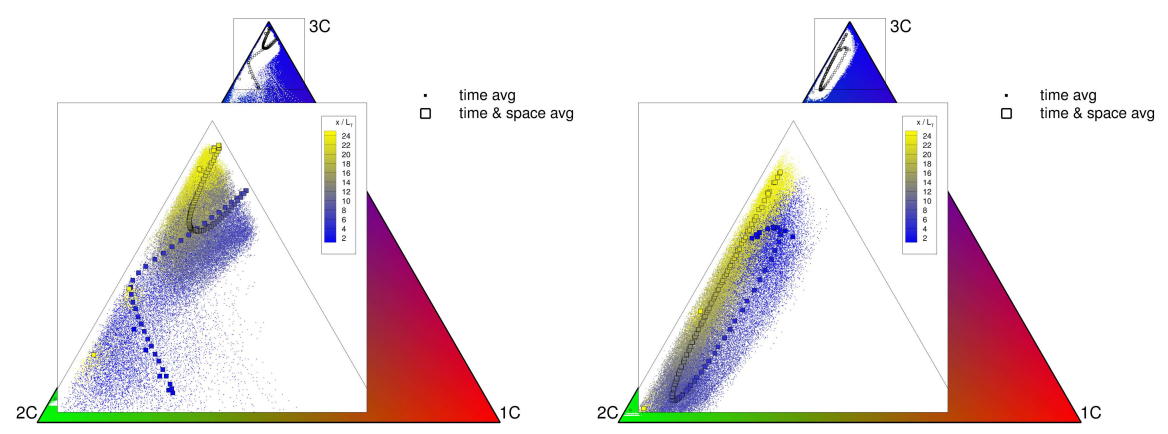

Fig. 3 Temporally and both temporally and spatially averaged decay of Reynolds stress anisotropy invariants for the original formulation (left) and periodic correction (right).

In the following, we will compare the development of the Reynolds stress tensor, its anisotropy invariants as well as two-point correlations and energy spectra. First insights into the deficiency of the original method in a periodic configuration can be gained from Fig. 2 (left). The thin lines represent the variation in temporal averages while the thick lines are temporally and spatially averaged. It can be observed that even in the temporal average, a spatial variation remains. The extreme values for the Reynolds stresses can be located at the periodic boundaries of the domain. If the periodic correction is applied, these spatial variations vanish and the temporal average at all locations converges to the spatial average. Furthermore, the spatial average of the $y$ - and $z$-fluctuating velocities do not show the spurious drop below the reference value of 1 as in the original formulation. Fig. 3 confirms these observations in terms of the turbulence anisotropy. The axial development within the barycentric map is colour-coded from blue at the inlet to yellow at the outlet. After a short distance of adaptation, the generated turbulence follows a return-to-isotropy path. With the correction, the scatter is greatly reduced.

Two-point correlations were gathered at various distances downstream of the inlet in both homogeneous directions. Fig. 4 shows them at an axial position $x / L_{T}=$ 

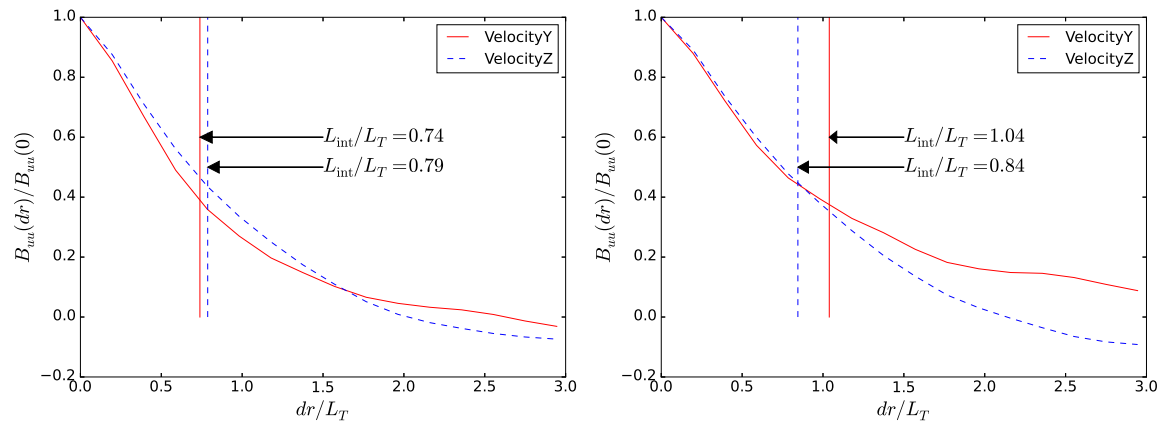

Fig. 4 Two-point correlations for $y$ - and $z$-velocity components at $x / L_{T}=1.5$ downstream of the inlet for the original formulation (left) and periodic correction (right).
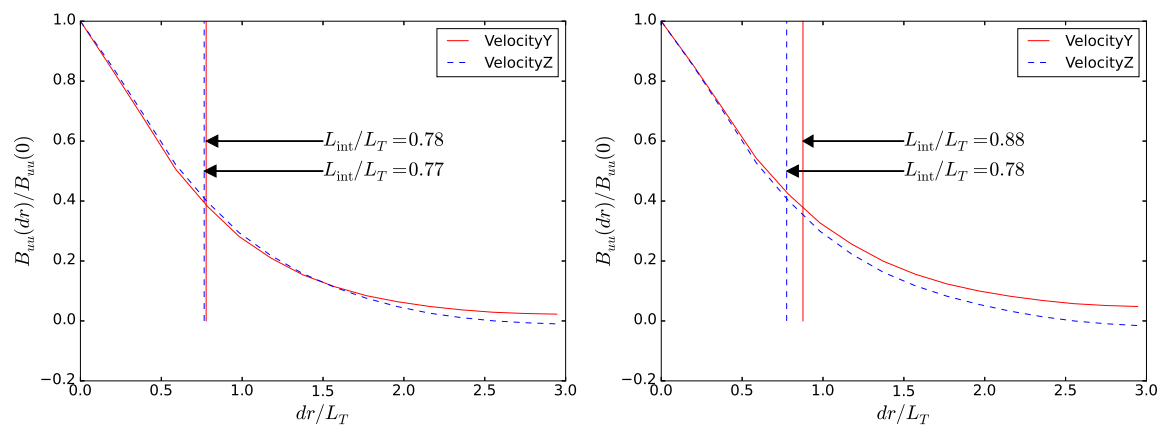

Fig. 5 Two-point correlations for $y$ - and $z$-velocity components at $x / L_{T}=14$ downstream of the inlet for the original formulation (left) and periodic correction (right).
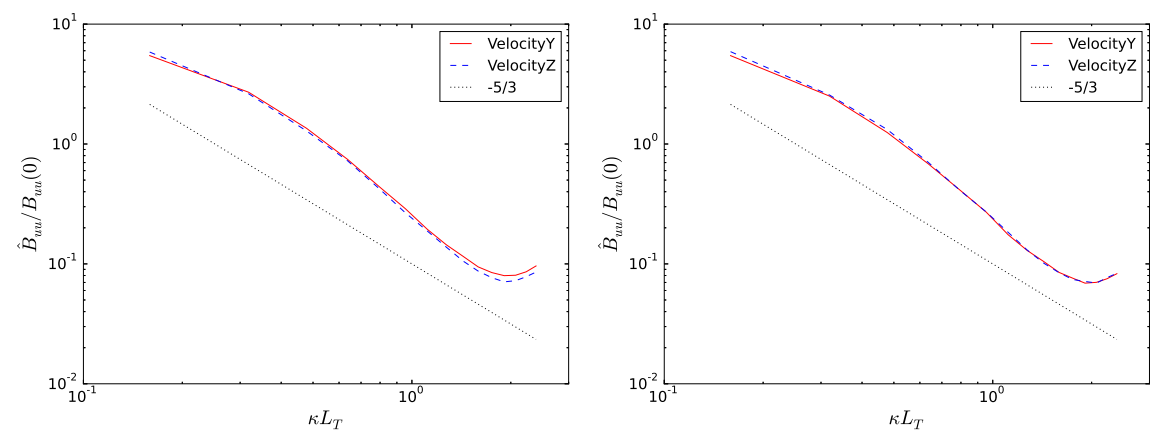

Fig. 6 Energy spectra for $y$ - and $z$-velocity components at $x / L_{T}=14$ downstream of the inlet for the original formulation (left) and periodic correction (right). 
1.5. In the original formulation (left), the length scales for $y$-and $z$-components of the velocity are nearly equal. The chosen algorithm clearly affects the length scale for the $y$-component of the velocity (right) whose two-point correlation does not reach 0 . Downstream, at $x / L_{T}=14$, this effect has already mixed out and both formulations show only very subtle differences in Fig. 5. From the two-point correlations at the latter station, one-dimensional energy spectra were computed by taking the Fourier transform. Fig. 6 shows that the periodic correction does not negatively affect the energy spectra.

\section{Conclusion}

We investigated the performance of a method to generate synthetic turbulence in the case of spatially decaying homogeneous isotropic turbulence. As this method showed spurious effects at the periodic domain boundaries, we suggested an approach to ensure periodicity. This approach eliminates the spatial variation of the temporal average. Analysis of the turbulence anisotropy as well as two-point statistics has shown that properties of the original method are preserved in the far field.

\section{References}

[1] K. Becker, K. Heitkamp, and E. Kügeler, "Recent progress in a hybrid-grid CFD solver for turbomachinery flows," in V European Conference on Computational Fluid Dynamics ECCOMAS CFD 2010, (Lisbon, Portugal), June 2010.

[2] A. Keating, U. Piomelli, E. Balaras, and H.-J. Kaltenbach, "A priori and a posteriori tests of inflow conditions for large-eddy simulation," Phys. Fluids, vol. 16, no. 12, pp. 4696-4712, 2004.

[3] B. van Leer, "Towards the ultimate conservative difference scheme. V. A second-order sequel to Godunov's method,' J. Comput. Phys., vol. 32, no. 1, pp. $101-136,1979$.

[4] P. L. Roe, "Approximate Riemann solvers, parameter vectors, and difference schemes," J. Comput. Phys., vol. 43, no. 2, pp. 357 - 372, 1981.

[5] M. L. Shur, P. R. Spalart, M. K. Strelets, and A. K. Travin, "Synthetic turbulence generators for RANS-LES interfaces in zonal simulations of aerodynamic and aeroacoustic problems," Flow Turbul. Combus., vol. 93, no. 1, pp. 63-92, 2014.

[6] J. Smagorinsky, "General circulation experiments with the primitive equations," in Monthly Weather Review, vol. 91, pp. 99-164, 1963.

[7] G. Tabor and M. Baba-Ahmadi, "Inlet conditions for large eddy simulation: A review," Comput. Fluids, vol. 39, no. 4, pp. 553 - 567, 2010. 